\title{
IMPACTO DE LA REDUNDANCIA ESTRUCTURAL EN EL COMPORTAMIENTO SÍSMICO DE ESTRUCTURAS DE CONCRETO REFORZADO
}

\author{
IMPACT OF STRUCTURAL REDUNDANCY ON THE SEISMIC BEHAVIOR OF \\ REINFORCED CONCRETE STRUCTURES
}

\author{
ARTURO TENA', JOSÉ CORTÉS², EBER GODÍNEZ ${ }^{3}$ \\ 1 Universidad Autónoma Metropolitana. México.tenac@servidor.unam.mx \\ 2 ICA Ingeniería. México.ingjosecortes@gmail.com \\ 3 Universidad Autónoma de Chiapas. México. eber.godinez@uncah.mx
}

RESUMEN

Se presentan los resultados de estudios paramétricos dedicados a evaluar el impacto que tiene el aumentar la redundancia estructural en edificios con base en marcos dúctiles de concreto reforzado (con y sin contravientos) de distintos niveles, al aumentar el número de crujias, utilizando análisis no lineales con carga monótonamente creciente "pushover", y así poder definir 0 evaluar numéricamente al factor de redundancia $(\rho)$.

Los edificios fueron analizados y diseñados como marcos dúctiles de concreto reforzado conforme a las recomendaciones del apéndice A de las Normas Técnicas Complementarias para Diseño por Sismo del Reglamento para las Construcciones del Distrito Federal vigente. Los marcos en estudio tienen las siguientes características: a) marcos con un mismo claro $\mathrm{L}=7 \mathrm{~m}$ y altura de entrepiso $\mathrm{H}=3.5 \mathrm{~m}$, con alturas de 4, 8, 12 y 16 niveles. Para cada marco antes descrito, se varió el número de crujías de 1, 2, 3 y 4, b) marcos con una altura de entrepiso $\mathrm{H}=3.5 \mathrm{~m}$ y una longitud total fija $\mathrm{L}_{\mathrm{TOT}}=12 \mathrm{~m}$. Los marcos son de 4 , 8,12 y 16 niveles, en los cuales se varió el número de crujias de 1,2,3 y 4 . Con base en los resultados del presente estudio y de estudios previos reportados en la literatura especializada, se concluye que, en aras de la transparencia en el diseño sismorresistente de marcos dúctiles de concreto reforzado (con 0 sin contravientos) y otros sistemas estructurales, sí se justifica que la redundancia estructural sea tomada en cuenta directamente en el diseño mediante un factor de reducción por redundancia $(\rho)$, como lo proponen y hacen normas estadounidenses como el ASCE-7 o IBC, y mexicanas como el Manual de Obras Civiles (MOC-2008), en lugar de que se siga asegurando falazmente que en el RCDF, la redundancia estructural es tomada en cuenta indirectamente con los factores de reducción por ductilidad y sobrerresistencia (sin estudios de por medio). Se demuestra que la redundancia afecta de distinta manera la capacidad de deformación y de resistencia, dependiendo las características del sistema estructural empleado.

PALABRAS CLAVE: redundancia estructural, ductilidad, sobrerresistencia.
ABSTRACT

The results of parametric studies are presented to evaluate the impact of increasing structural redundancy in buildings based on ductile frames of reinforced concrete (with and without diagonal bracings) of different levels, increasing the number of beams using nonlinear analysis with monotonically increasing load "pushover", and thus to be able to define or evaluate numerically the redundancy factor ( $\rho$ ).

The buildings were analyzed and designed as ductile reinforced concrete frames in accordance with the existing recommendations of appendix A of the Complementary Technical Standards for Earthquake Design of the Regulations for the Constructions of the Federal District. The frames under study have the following characteristics: a) frames with the same span $\mathrm{L}=$ $7 \mathrm{~m}$ and mezzanine height $\mathrm{H}=3.5 \mathrm{~m}$, with heights of 4, 8, 12 and 16 levels. For each frame described above, the number of crossings of 1, 2, 3 and 4 was varied, b) frames with a mezzanine height $\mathrm{H}=3.5 \mathrm{~m}$ and a fixed total length LTOT = $12 \mathrm{~m}$. The frames were 4, 8, 12 and 16 levels, in which the number of beams of 1, 2, 3 and 4 were varied. Based on the results of the present study and previous studies reported in the specialized literature, it concludes that, in the interests of transparency in the seismic resistant design of reinforced concrete ductile frames (with or without diagonal bracings) and other structural systems, it is justified that structural redundancy is directly taken into account in the design by a reduction factor by Redundancy ( $\rho$ ), as proposed and made by US standards such as ASCE-7 or IBC, and Mexican as the Manual of Civil Works (MOC-2008), rather than continue to fall into the assumption that in the RCDF, structural redundancy Is taken into account indirectly with the factors of reduction by ductility and over-resistance (without studies of means). It is shown that the redundancy affects differently the deformation capacity and resistance, depending on the characteristics of the structural system used.

KEYWORDS: structural redundancy, ductility, over-resistance. 
INTRODUCCIÓN

En la actualidad, las demandas y tendencias arquitectónicas responden a establecer grandes espacios, necesarios para la optimización de áreas de construcción, el alto costo de los predios en las zonas financieras o de negocios de las ciudades y las malas estructuraciones por parte de los arquitectos e ingenieros, en especial en edificios con base en marcos rígidos. Esto ha provocado que se construyan edificios con grandes claros y muy pocas crujías en alguna dirección, lo que redunda en estructuras con un grado de hiperestaticidad relativamente bajo.

Las experiencias que han dejado los sismos pasados, estudios experimentales y analíticos, señalan que la ductilidad y redundancia estructural han resultado ser los medios más efectivos para proporcionar seguridad contra el colapso y daños excesivos, especialmente si los movimientos resultan más severos que los anticipados por el diseño, pues en el momento en que sucede un sismo de gran intensidad, la respuesta global de la estructura deja de ser lineal e incursiona en su intervalo no lineal, lo que produce irremediablemente algún tipo de daño en elementos. Debido a que el diseño sismorresistente contempla la posibilidad de daño en algunos elementos estructurales durante la ocurrencia de algún sismo fuerte, la estructura debe ser capaz de acomodar todo este daño sin experimentar colapso.

Un buen detallado en una estructura de concreto reforzado aumenta su capacidad de de-
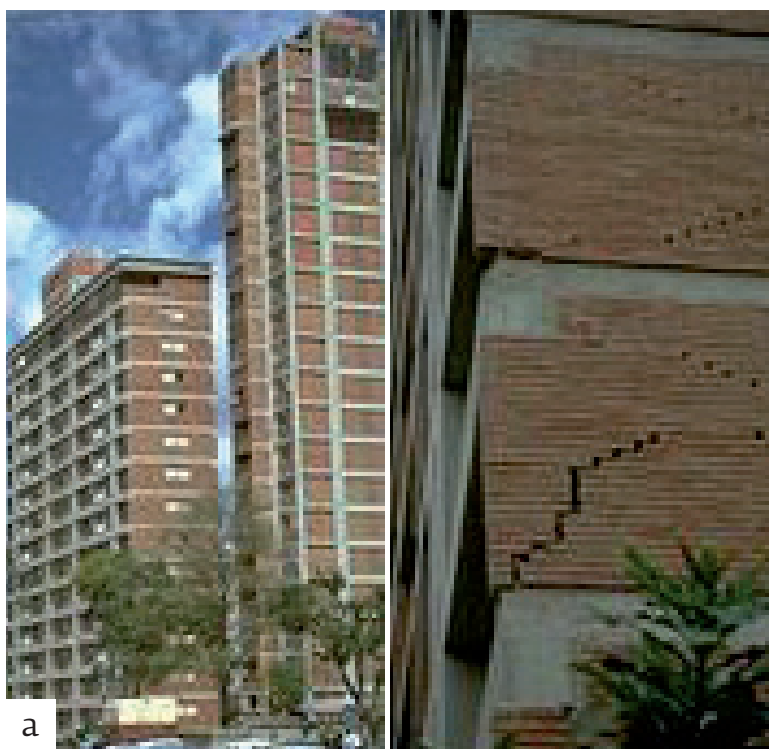

formación y le permite desarrollar la máxima resistencia de sus elementos, hasta que éstos no pueden aportar más rigidez ni resistencia y se formen articulaciones plásticas. El número de articulaciones plásticas necesarias para causar la inestabilidad de la estructura, y por consiguiente el colapso, depende de la redundancia de la estructura. Por lo tanto, el grado de redundancia aumenta la capacidad de la estructura para incursionar en el intervalo no lineal. Esto se debe a que en la estructura se redistribuyen las solicitaciones de los elementos más demandados, o elementos que entraron en su intervalo no lineal por la formación de articulaciones plásticas, a elementos con menor solicitación.

La redundancia se refiere a la existencia de una gran cantidad de líneas de defensa continuas y resistentes a la carga, que causa un alto grado de hiperestaticidad. Una de las características más importantes que se debería buscar en una estructura, desde el punto de vista de diseño sísmico, es la redundancia, ya que cuando se cuenta con un número reducido de elementos, como es el caso de estructuras poco redundantes, la falla de alguno de éstos puede causar el colapso de la estructura. Por ello, un buen diseño sismorresistente debe tratar de distribuir las cargas laterales producidas por terremotos de gran intensidad entre el mayor número de elementos posibles, para permitirlea la estructura desarrollar su máxima resistencia y aprovechar la disipación de energía producida por histéresis.

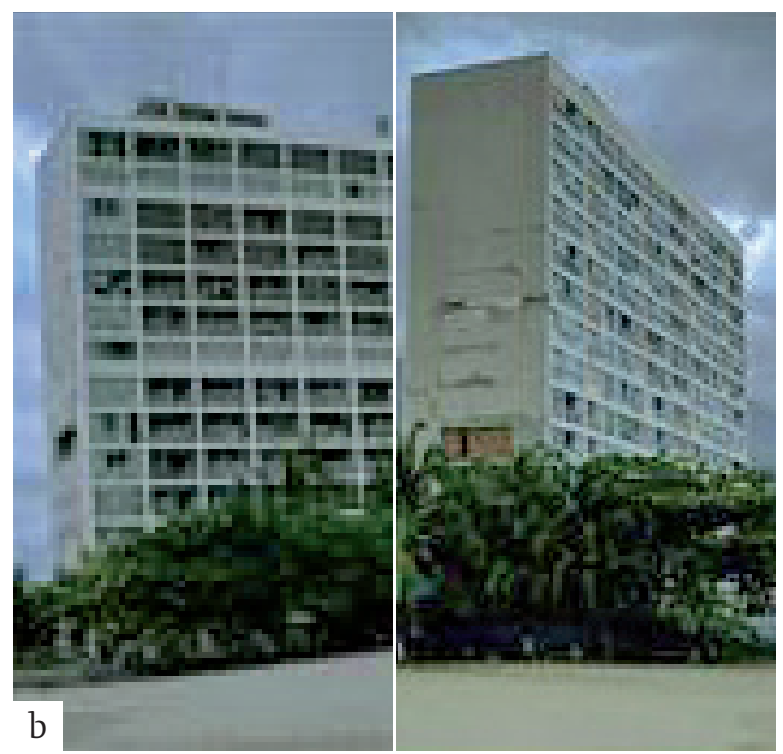

Figura 1. Edificios poco redundantes en la dirección corta que presentaron daño estructural durante el sismo de Caracas (fotos de la colección de Karl Steinbrugge, Web-Berkeley 2010).

a) Edificios Petunia, con una línea de b) Edificio Laguna Beach, con marco de una crujía defensa (muros) en la dirección con muros diafragma en la dirección corta, corta, esbeltos y planta alargada esbelto y con planta alargada. 
El olvido de la importancia del concepto de redundancia ha fomentado el uso de estructuraciones vulnerables en edificios urbanos, donde en muchas ocasiones se estructura en una dirección corta marcos con una sola crujía, lo que favorece además dos condiciones de irregular estructura: plantas alargadas y esbeltez. Ejemplos de lo anterior se han dado varios, por citar algunos casos, los edificios Petunia (figura 1a) y Laguna Beach (figura 1b) durante el sismo de Caracas del 29 de julio de 1967 (Web Berkeley 2010, Tena 2010); el multifamiliar Juárez durante el sismo de Michoacán del 19 de septiembre de 1985, que era un conjunto de edificios de considerable altura que además contaban con plantas alargadas y esbeltas (figura 2a), y cuya estructuración en la dirección esbelta, donde finalmente colapsó (figura $2 \mathrm{~b}$ ), era poco redundante (figura 2a).

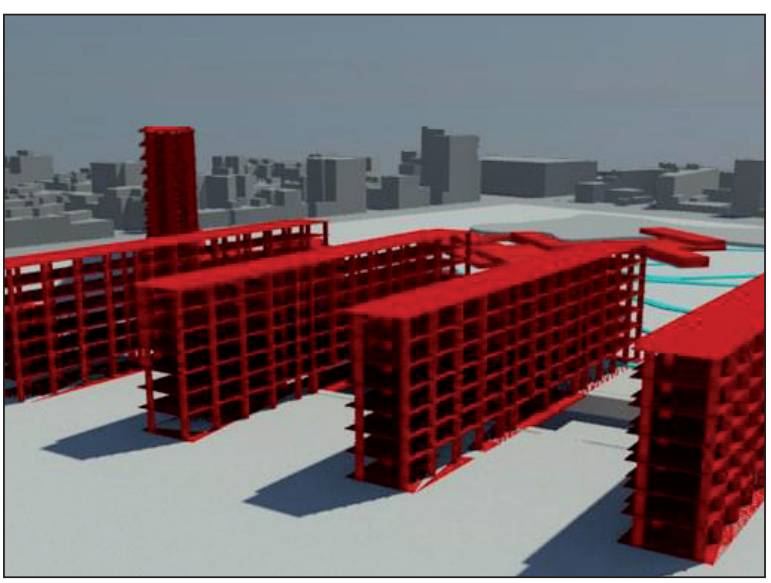

a) Estructuración de los multifamiliares

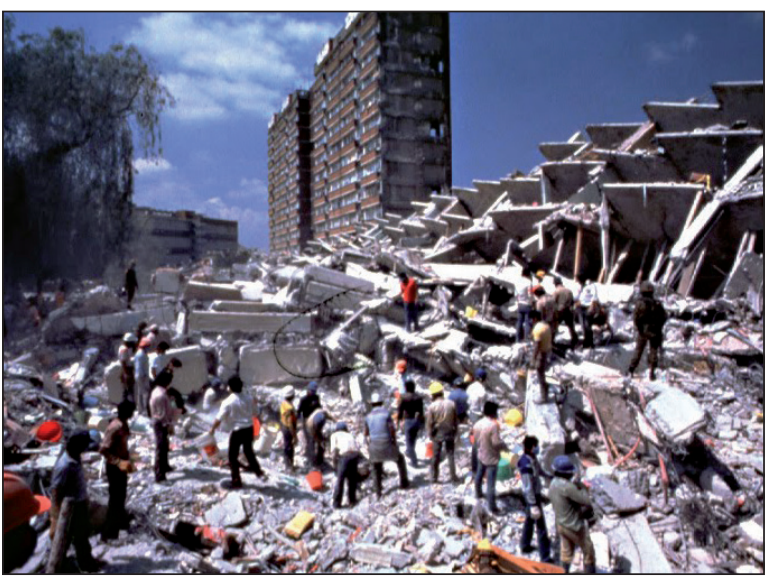

b) Colapso del Edificio C-4 Juárez

Figura 2. Colapso del edificio C-4 del multifamiliar Juárez durante sismo del 19 de septiembre de 1985 (fotos e imágenes de http://www.arqred.mx/blog/ tag/multifamiliar-juarez).

Con base en lo anteriormente ilustrado, se considera que sí se debe tomar en cuenta de manera transparente la redundancia estructural en el diseño sísmico de edificios, pues en sismos anteriores se ha demostrado que estas estructuras son vulnerables, no importando que sean de poca altura, o de mediana a gran altura (figuras 1 y 2).

\section{DEFINICIÓN DE REDUNDANCIA ANTE CARGA LATERAL}

La definición de redundancia ante cualquier patrón de carga se describe en función del número de regiones críticas, llamadas articulaciones plásticas, necesarias para causar la inestabilidad o colapso de la estructura.

La definición de redundancia ante carga laterales como las producidas por los sismos se describe en función del número de articulaciones plásticas necesarias para causar la inestabilidad o colapso de la estructura bajo la acción de cargas laterales y gravitacionales. En este caso, el grado de redundancia ante la carga lateral no sólo depende de las propiedades geométricas estructurales, refuerzo y detallado, sino también del comportamiento dinámico de la estructura, y del patrón de cargas laterales (Bertero y Bertero 1999).

En las figuras 3a y $3 \mathrm{~d}$ se presentan dos marcos con el mismo grado de indeterminación estática $\left(n_{e}=6\right)$. En las figuras $3 b, 3 c$, 3e y $3 f$ se representan los mecanismos de colapso teóricos que definen el grado de indeterminación ante cargas sísmicas, $\mathrm{n}_{\mathrm{s}}$. En las figuras $3 \mathrm{~b}$ y 3 e se muestran los mecanismos de colapsos deseados reglamentariamente, los que pueden obtenerse con un buen detallado estructural que favorezca el mecanismo de viga débil - columna fuerte. En las figuras 3 c y $3 f$ se ilustran mecanismos de colapso indeseables por primer piso suave. Lo que cabe destacar es demostrar que, conforme a la teoría, un marco con menos crujías es siempre menos redundante ante carga lateral que un marco que tenga el mismo grado de indeterminación estática, pero más crujías. Se aprecia que el marco de la figura 3a, de dos crujías, siempre tiene una mayor redundancia ante carga lateral con respecto al marco de una crujía de la figura 3d, ya sea que el mecanismo de colapso corresponda a viga débil - columna fuerte $\left(n_{s}=7\right.$ vs $\left.n_{s}=6\right)$ o a un piso suave $\left(\mathrm{n}_{\mathrm{s}}=6\right.$ vs $\left.\mathrm{n}_{\mathrm{s}}=4\right)$.

Bertero y Bertero (1999) enfatizan que la redundancia produce varios efectos benéficos sobre la respuesta estructural como: a) evita la concentración de daño al aumentar la disipación de energía entre varios elementos, b) disminuye los desplazamientos laterales y la demanda de ductilidad debida a torsión inelástica y, c) permite la obtención de elementos menos robustos 


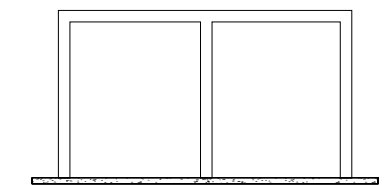

a) $\mathbf{n}_{\mathbf{e}}=6$

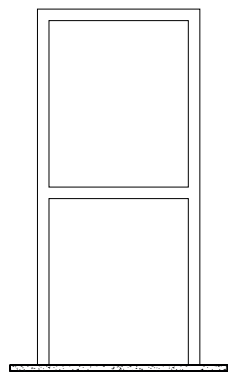

d) $n_{e}=6$
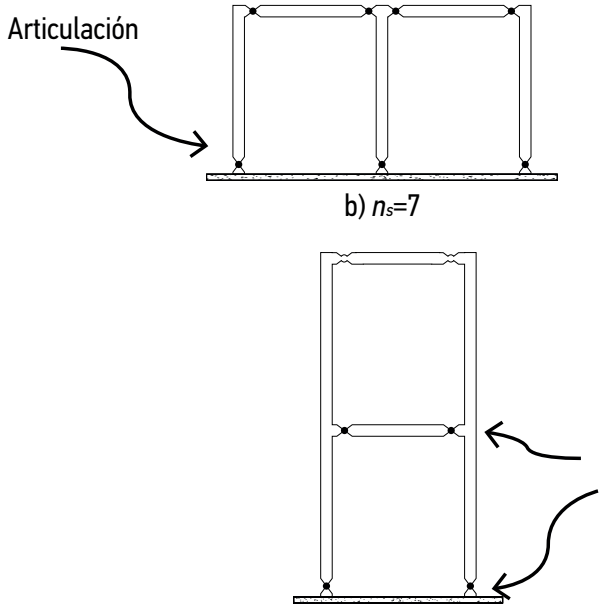

e) $n_{s}=6$
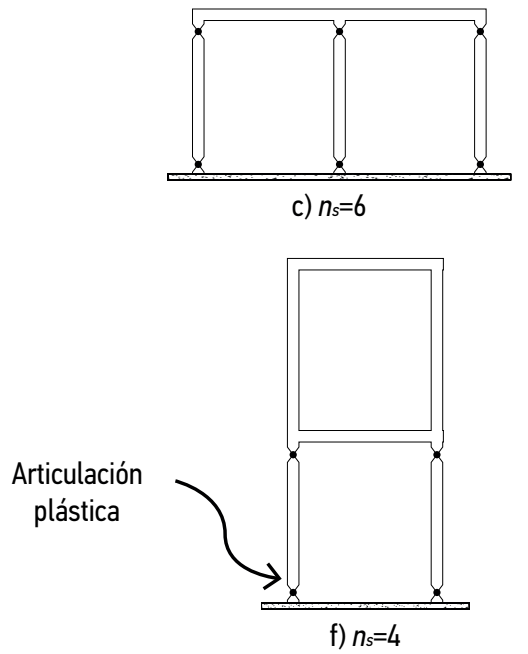

Figura 3. Diferencias entre indeterminación estática y redundancia ante cargas laterales (adaptado de Bertero y Bertero, 1999)

en comparación a edificios menos redundantes. Por lo anterior, cada uno de estos efectos debería ser considerado en el lugar correspondiente en el diseño sísmico, con un factor de redundancia, y no debería ser incluido de manera indirecta en otros factores.

Estas observaciones generales que realizaron en su estudio Bertero y Bertero (1999) pueden ser ilustradas con modelos sencillos de marcos de una y dos crujías (figura 4) analizados al límite (análisis plástico) y considerando condiciones ideales. En los resultados del análisis se observa que al agregar una crujía extra, la capacidad de carga del marco aumenta 54.5\%. Además de aumentar su capacidad de incursionar en su intervalo no lineal por la formación de una mayor cantidad de articulaciones plásticas, en estructuras reales esto aumenta la disipación de energía por histéresis, evita la acumulación de daño en los elementos y disminuye la demanda de ductilidad debida a torsión inelástica. Ahora, si consideramos que los elementos de los dos marcos tienen las mismas secciones al considerar que el momento plástico $M_{p}$ en las dos estructuras es idéntico, se puede justificar reducir las secciones del marco de la figura $4 \mathrm{~b}$ para obtener una capacidad de carga semejante a la del marco de la figura 4a, lo que demuestra que se pueden obtener secciones menos robustas en estructuras más redundantes.

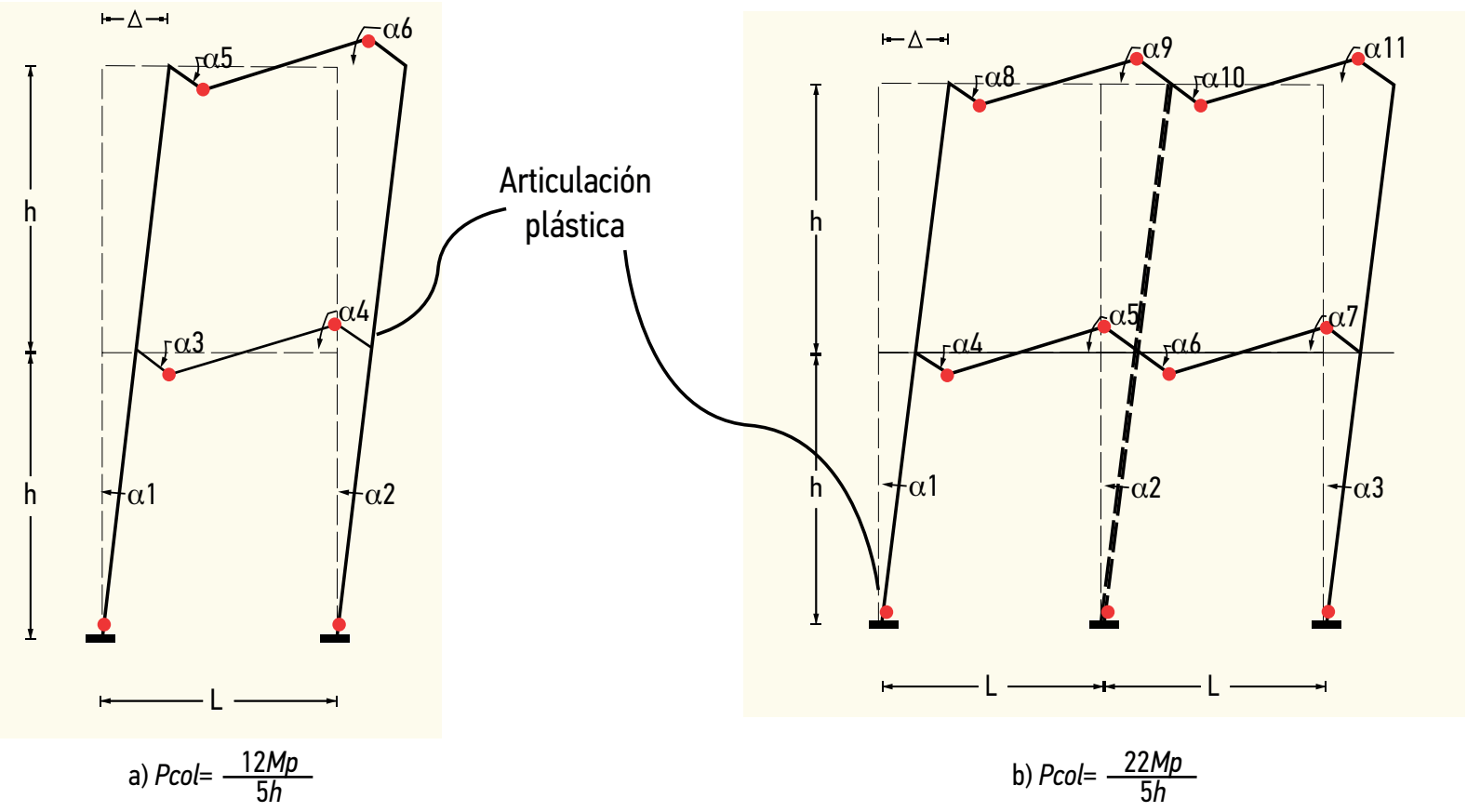

Figura 4. Análisis plástico de marcos de una y dos crujías. 


\section{INVESTIGACIÓN PREVIA}

A pesar de su importancia en el desempeño de las estructuras ante sismos intensos, la redundancia estructural ha sido relativamente poco estudiada. La mayor parte de los estudios disponibles tienen un enfoque probabilista (Feng y Moses 1986, Frangopol y Curley 1987, Fu y Frangopol 1990, Paliou et al. 1990, Bertero y Bertero 1999, Song y Wen 2000, Fallah et al. 2009) o mixto (Bertero y Bertero 1999, Whittaker et al. 1999, Husain y Tsopelas 2004, Tsopelas y Husain 2004), o exclusivamente determinista (Tena-Colunga 2004). De los pocos estudios recientes disponibles, los investigadores que los realizaron no están de acuerdo en combinar sus efectos con otras variables, como se hace hasta la fecha en algunos reglamentos vigentes (por ejemplo, Bertero y Bertero 1999, Tsopelas y Husain 2004). Está fuera del alcance de este estudio hacer una revisión bibliográfica exhaustiva, la cual se reporta en trabajos previos de los autores (Cortés 2012, Tena y Cortés 2014 y 2015, Godínez y Tena 2015).

\section{REGLAMENTACIÓN VIGENTE}

Hasta donde saben los autores, la primera propuesta reglamentaria para introducir un factor de reducción por redundancia ( $\rho$ ) fue hecha por el reglamento UBC-97 (1997) y desde ese entonces el concepto ha evolucionado en las recomendaciones de diseño sísmico más recientes de los Estados Unidos (ASCE-7 2010).

ASCE-7

En el documento ASCE-7 (2010), el factor de corrección por redundancia estructural, $\rho$, se toma en cuenta en las combinaciones de carga utilizadas durante el análisis de la estructura mediante la consideración de los efectos sísmi$\cos \mathrm{E}$ :

$$
\begin{aligned}
& E=\rho Q_{E}+0.2 S_{d S} D \\
& E=\rho Q_{E}-0.2 S_{d S} D
\end{aligned}
$$

donde $E$ es el efecto combinado de fuerzas horizontales y verticales inducidas por el sismo; D es el efecto de la carga muerta, $Q_{E}$ es el efecto de la fuerza sísmica horizontal y SDS es la aceleración del espectro de diseño para periodos cortos.

Se tomará $\rho=1.0$ cuando se cumplan las siguientes características:

Estructuras asignadas a la categoría de diseño sísmico B o C
- En el cálculo de distorsiones de entrepiso $y$ efectos P- $\Delta$

- Diseño de componentes no estructurales

- Diseño de estructuras que no sean similares a edificios

- Cargas de diseño de diafragmas

- Estructuras con sistemas de amortiguamiento

Para estructuras asignadas a la categoría de diseño $\mathrm{D}, \mathrm{E}$ o $\mathrm{F}, \rho=1.3$, a no ser que una de las condiciones siguientes se cumpla, por lo cual se permite que $\rho=1.0$ :

a. Estructuras que son regulares en planta y elevación que son provistas con un sistema de resistencia ante carga lateral que consiste de al menos dos crujías perimetrales en cada lado de la estructura y en cada dirección ortogonal de cada entrepiso. El número de crujías para muros de cortante va a ser calculado como la longitud del muro dividido por la altura de entrepiso o dos veces la longitud del muro dividido entre la altura de entrepiso para estructuras de marcos ligeros.

b. Cada entrepiso de la estructura resiste más del 35\% del cortante basal.

En el ASCE-7 con el factor $\rho$ se toma en cuenta el riesgo sísmico de la zona donde se construirá la estructura, que se considera en la asignación de la categoría de diseño sísmico, ya sea por periodos cortos (SDS) o periodos de un segundo (S1). Además, considera la importancia de la construcción, castigando a estructuras que necesitan estar en funcionamiento después de algún sismo o desastre natural; esto se hace considerando el uso de la estructura en la asignación de las categorías de diseño sísmico. Por último, con el factor $\rho$ se castiga a estructuras donde gran parte de su resistencia depende de un solo elemento, por ejemplo, cuando un elemento proporciona más del 33\% de la resistencia de un entrepiso.

\section{MOC-2008}

El Manual de Obras Civiles (MOC-2008 2009) de la Comisión Federal de Electricidad es la primera normatividad de México en introducir un factor de reducción por redundancia (MOC-2008 2009, Tena-Colunga et al. 2009), pero la manera en que se toma en cuenta es distinta a como lo 
hacen los reglamentos de Estados Unidos referidos anteriormente.

En el MOC-2008 (2009) se proponen modificar las aceleraciones del espectro de diseño, además de en función de la ductilidad ( $\left.Q^{\prime}\right)$ y la sobrerresistencia (R), por la redundancia ( $\rho$ ) del sistema estructural (figura 5):

$$
a^{\prime}=\frac{a(\beta)}{Q^{\prime} R \rho}
$$
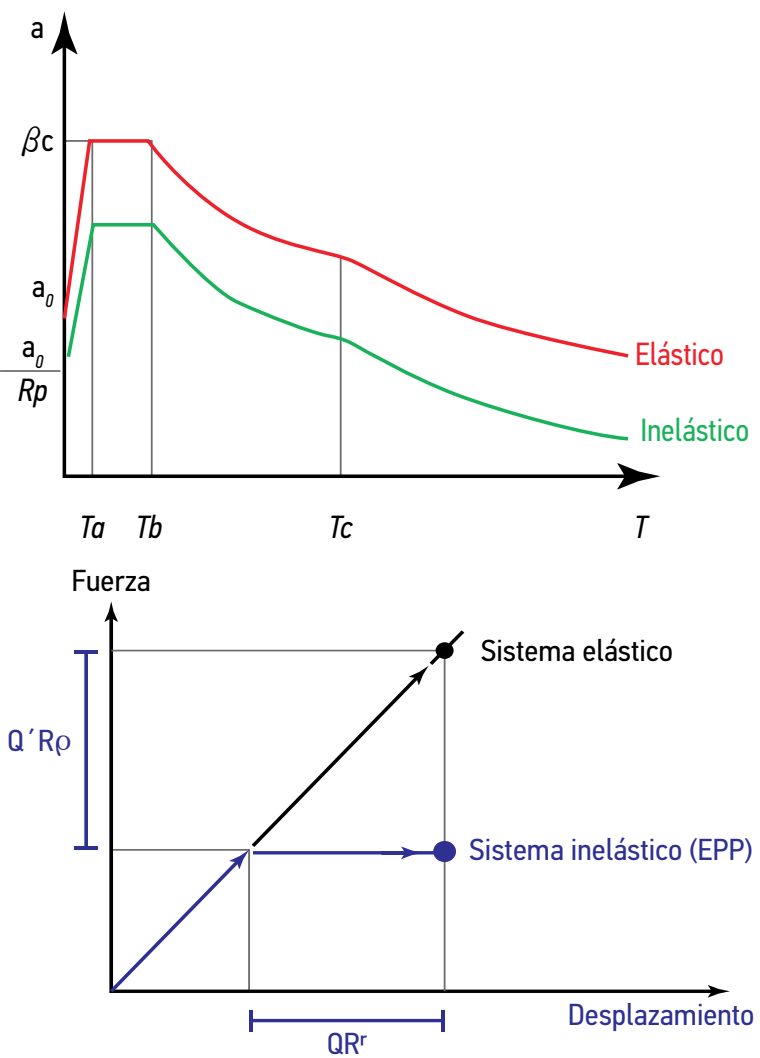

Figura 5. Reducción del espectro elástico de diseño del MOC-2008 por concepto de ductilidad $\left(Q^{\prime}\right)$, sobrerresistencia (R) y redundancia $(\rho)$.

El factor de redundancia, $\rho$, se toma en cuenta para cada dirección ortogonal de análisis de la manera siguiente:

$\rho=0.8$ en estructuras con al menos dos marcos o líneas de defensa paralelas en la dirección de análisis, cuando se disponga de marcos de una sola crujía o estructuraciones equivalentes (un solo muro por línea de defensa paralela, etc.).

$\rho=1.0$ en estructuras con al menos dos marcos o líneas de defensa paralelas en la dirección de análisis y que cada marco o línea de defensa disponga de al menos dos crujías o estructuraciones equivalentes.

$\rho=1.25$ en estructuras con al menos tres marcos o líneas de defensa paralelas en la di- rección de análisis y que cada marco o línea de defensa disponga de al menos tres crujías o estructuraciones equivalentes.

Con la introducción del factor de redundancia $\rho$ en el MOC-2008, se reconoce directamente que la estabilidad, la capacidad de deformación y la resistencia adicional de las estructuras aumentan a medida de que éstas son más redundantes. El propósito de $\rho$ es premiar con reducciones mayores el diseño de estructuras más redundantes (de mejor comportamiento) y castigar o desalentar el diseño de los sistemas menos redundantes, de comportamiento más incierto, propensos a concentrar las deformaciones inelásticas y, con ello, a desarrollar menor ductilidad y/o resistencia adicional.

La propuesta de corrección por redundancia considera que ésta ya ha sido de alguna manera tomada en cuenta indirectamente en los valores del factor de comportamiento sísmico $Q$ y del factor de reducción por sobrerresistencia, $R$, por lo que se requieren hacer ajustes para los sistemas más redundantes y menos redundantes. En todos los casos, se enfatiza que cualquier estructura debe poseer al menos dos marcos o líneas de defensa paralelas en la dirección de análisis, por lo que no será aceptable estructurar edificios con una sola línea de defensa en la dirección de análisis, como pudiera ser, por ejemplo, una estructuración tipo péndulo invertido.

Las estructuraciones donde se propone $\rho=1.0$ corresponden esencialmente a aquellas con la tipología más generalmente utilizada en estudios en los que se basan algunas de las propuestas de los factores de reducción por sobrerresistencia. Se solicita además un mínimo de dos crujías por marco, con base en los resultados de los estudios presentados por Husain y Tsopelas (2004), Tsopelas y Husain (2004) y Tena-Colunga (2004). Se propone $\rho=1.25$ para sistemas redundantes, con base en estudios relativamente recientes, donde se ha comprobado que este tipo de sistemas son capaces de desarrollar demandas de deformación y factores de sobrerresistencia mayores a los propuestos en su diseño (Tena-Colunga et al. 2008). Se propone $\rho=0.8$ para los sistemas poco redundantes, como los marcos de una sola crujía tomando en cuenta lo siguiente: (a) la vulnerabilidad de este tipo de estructuraciones manifestada en sismos anteriores, particularmente si tienden a ser esbeltas, como se ilustró en la introducción, (b) esta estructuración tiende a concentrar la plasticidad y a que se formen mecanismos frágiles de piso débiles o mixtos 
con plasticidad concentrada en columnas (por ejemplo, Tena-Colunga 2004) y, (c) algunos estudios conducidos en marcos de concreto reforzado de una sola crujía para estimar factores de sobrerresistencia han reportado valores de $\mathrm{R}=1.5 \mathrm{o}$ inferiores.

\section{ALCANCE DEL PROYECTO DE INVESTIGACIÓN}

Se reporta con mayor detalle en Cortés (2012), Tena y Cortés (2014 y 2015) y Codínez y Tena (2015) es determinar el impacto en el factor de redundancia ( $\rho$ ) de aumentar el número de crujías en edificios con marcos dúctiles de concreto reforzado de distintos niveles, con y sin contravientos, utilizando análisis no lineales con carga monótonamente creciente (pushover), y así poder valorar qué tan acertada o no es la propuesta del MOC-2008 (2009), pues como se señala en los comentarios de dicho documento: «Aunque la propuesta se basa en los resultados de varios estudios, también está basada en la intuición y la experiencia, por lo que es susceptible de revisión y de mejoras que se verán reflejadas en futuras revisiones de este cuerpo normativo».

\section{MODELOS ESTRUCTURALES}

Para alcanzar los objetivos planteados en este trabajo de investigación, el primer paso consistió en diseñar edificios con base en marcos dúctiles de concreto reforzado regulares en planta y elevación ante un cortante basal $\mathrm{V} /$ $W=0.1$. El diseño se realizó conforme a las Normas Técnicas Complementarias para Diseño de Estructuras de Concreto vigentes en México (NTCC-04 2004). Los marcos en estudio tienen las siguientes características:

- Marcos dúctiles de concreto reforzado con una altura de entrepiso $\mathrm{H}=3.5 \mathrm{~m}$ y una longitud total fija $\mathrm{L}_{\mathrm{TOT}}=12 \mathrm{~m}$ (figura 6), con alturas de 4, 8, 12 y 16 niveles en los cuales se varió el número de crujías: 12,3 y 4.

- Marcos dúctiles de concreto reforzado con contravientos chevrón con una altura de entrepiso $\mathrm{H}=3.5 \mathrm{~m}$ y una longitud total fija $\mathrm{L}_{\mathrm{TOT}}=12 \mathrm{~m}$, con alturas de 4, 8, 12 y 16 niveles en los cuales se varió el número de crujías: 12 , 3 y 4. Para los modelos de tres y cuatro crujías se realizó el diseño y evaluación de dos casos: a) modelos en que todas las crujías del marco están contraventeadas (identificados con el sufijo V1) y, b) modelos en los que única- mente están contraventeadas las crujías exteriores del marco (figura 7).

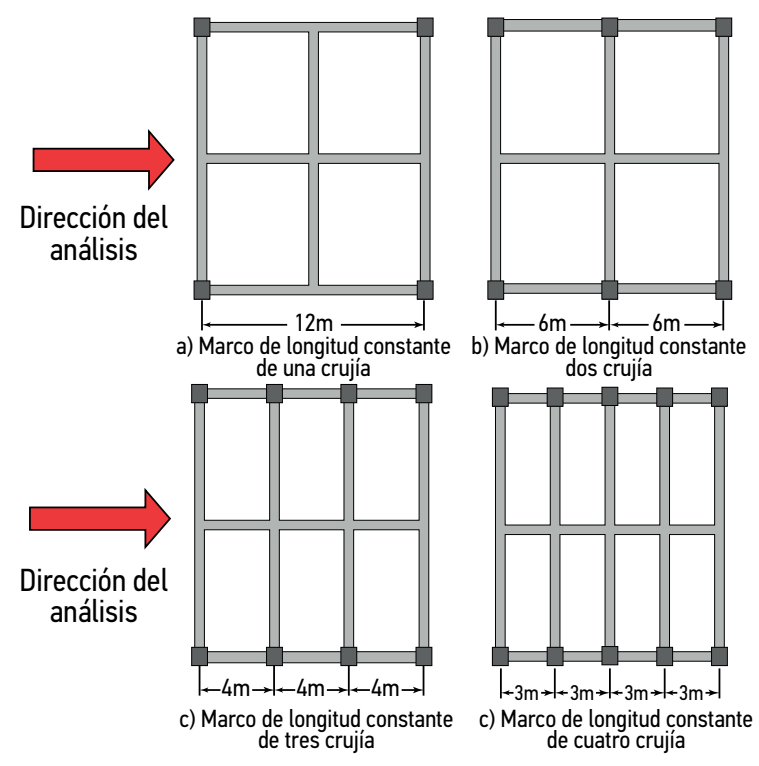

Figura 6. Planta tipo de los edificios diseñados con una longitud total fija $\mathrm{L}_{\mathrm{TOT}}=12 \mathrm{~m}$.

Las cargas vivas máximas e instantáneas de diseño se tomaron de las Normas Técnicas Complementarias sobre Criterios y Acciones para el Diseño Estructural de las Edificaciones (NTCCA04 2004). En entrepisos se utilizaron las cargas correspondientes a uso de oficinas y en el último nivel se tomaron las cargas de azoteas con pendiente no mayor a 5\%. Para las cargas muertas se cuantificó el peso de cada elemento estructural y no estructural que comúnmente se encuentra actuando en una oficina. Las cargas se supusieron uniformemente distribuidas sobre la losa. En el análisis y diseño de los marcos se consideraron las combinaciones de carga permanentes 1.4 $\left(\mathrm{C}_{\text {MUE }}+\mathrm{W}_{\text {MAX }}\right)$ y acciones accidentales 1.1 $\left(\mathrm{C}_{\mathrm{MUE}}+\mathrm{W}_{\mathrm{INS}}\right)$.

Dado que por simplicidad para la comparación de la respuesta de los distintos modelos se fijó el cortante basal de diseño al 10\% del peso total del edificio $(\mathrm{V}=0.10 \mathrm{~W})$, los edificios se diseñaron mediante el método estático conforme a las NTCS-04 (2004), el cual toma en cuenta la participación de modos superiores en el patrón de carga lateral para estructuras de periodo largo (en la rama descendente del espectro). Conforme al método estático de las NTCS-04, la distribución de fuerzas laterales se hace de la siguiente manera:

$$
\begin{aligned}
F_{i} & =V_{B} \frac{W_{i} h_{i}}{\sum W_{i} h_{i}} ; s i T \leq T_{b} \\
F_{i} & =W_{i}\left(k_{1} h_{1}+k_{2} h_{i}^{2}\right) \frac{a}{Q^{\prime}} ; s i T>T_{b}
\end{aligned}
$$




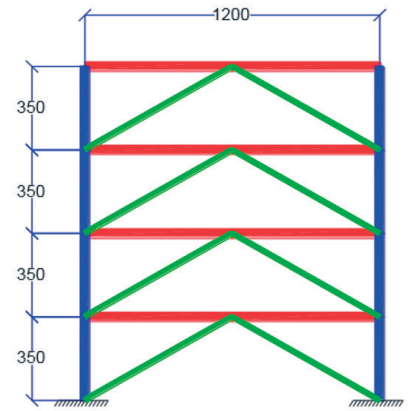

A) M1C4N

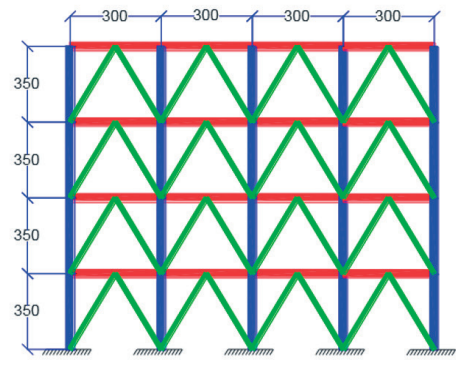

A) $M 4 C 4 N-V 1$

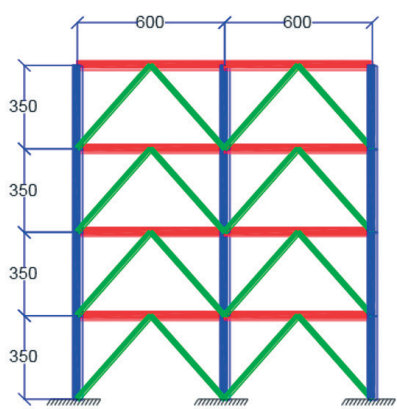

A) $\mathrm{M} 2 \mathrm{C} 4 \mathrm{~N}$

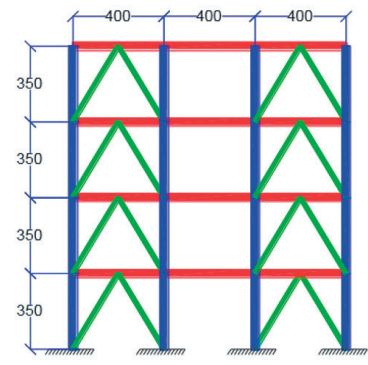

A) $\mathrm{M} 3 \mathrm{C} 4 \mathrm{~N}$

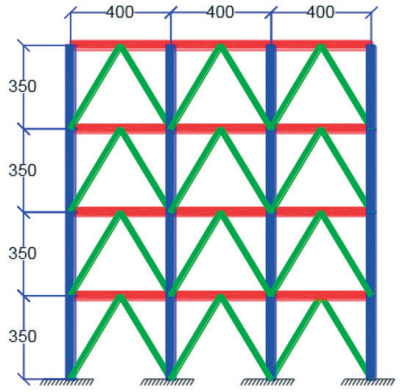

A) M3C4N-V1

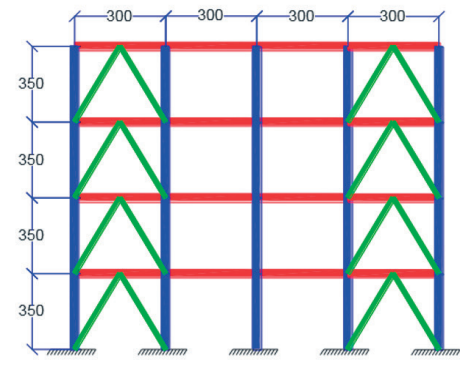

A) $M 4 C 4 N$

Figura 7. Elevación y nomenclatura de los modelos con contravientos en estudio.

$$
\begin{aligned}
& k_{1}=[1-0.5 r(1-q)] \frac{\sum W_{i}}{\sum W_{i} h_{i}^{2}} \\
& k_{2}=0.75 r(1-q) \frac{\sum W_{i}}{\sum W i h_{i}^{2}}
\end{aligned}
$$

donde $F_{i}, W_{i} y h_{i}$ son la fuerza lateral, el peso y la altura del i-ésimo nivel de la estructura sobre el terreno, respectivamente, $T$ es el periodo de la estructura y $\mathrm{T}_{b}$ es el periodo característico que define el final de la meseta y el inicio de la rama descendente del espectro de diseño.

Los análisis de los modelos se hicieron con programas comerciales de análisis estructural bajo las siguientes hipótesis: 1) un empotramiento perfecto en la base de las columnas de todos los modelos, 2) se consideraron las inercias brutas de las secciones vigas y columnas, por las razones que se explican con detalle en otros trabajos (Tena-Colunga et al. 2008 y Tena et al. 2012), 3) una rigidez infinita de los nudos viga-columna igual al $50 \%$ de sus dimensiones nominales, 4) el concreto tiene las siguientes propiedades índice: $\mathrm{f}^{\prime} \mathrm{C}=250 \mathrm{~kg} / \mathrm{cm} \mathrm{y,} \mathrm{5)} \mathrm{el}$ acero de los contravientos tiene las siguientes propiedades índice: $\mathrm{fy}=2530 \mathrm{~kg} / \mathrm{cm} 2$, Es $=2 \times 106$ $\mathrm{kg} / \mathrm{cm} 2$ y v=0.3.

En el diseño de los marcos dúctiles sin contravientos se intentó llegar a la distorsión de entrepiso límite de $0.03(3 \%)$ que se establece en el Apéndice A de las NTCS-04 (2004). Las distorsiones de entrepiso de diseño se obtuvieron multiplicando las distorsiones obtenidas de los análisis elásticos, por el factor de comportamiento sísmico $(\mathrm{Q}=4)$ y por el factor de sobrerresistencia (R) correspondientes según las mismas NTCS-04. Las envolventes de distorsiones de entrepiso de diseño se presentan con detalle en Cortés (2012). Las distorsiones de entrepiso máximas de diseño para cada modelo se resumen en la tabla 1 , donde se aprecia que las distorsiones máximas de diseño de los modelos de 4 niveles son las más bajas, debido a que en estos modelos rigieron el diseño de muchos elementos las combinaciones de cargas verticales. En general, las distorsiones de diseño más altas se obtuvieron para los modelos menos redundantes (con menos crujías).

Los diseños de los modelos se realizaron siguiendo las disposiciones para marcos dúctiles que establecen de las NTCC-04 (2004). El diseño se hizo siguiendo los pasos establecidos en las NTCC-04 para obtener un mecanismo de falla columna fuerte-viga débil conforme a principios de diseño por capacidad. Para fines de diseño, se consideró el cambio de las secciones de vigas y columnas como se indica esquemáticamente con colores en la figura 8 . Se distinguió además en el diseño (refuerzos) a columnas exteriores de interiores y a vigas exteriores de interiores. Los detalles de los diseños (secciones, armados, detallado, capacidades nominales, etc.) se presentan en Cortés (2012). En la tabla 1 se presentan los intervalos en los que variaron los cuantías de refuerzo a flexión 
TABLA 1. RESUMEN DE LOS DISEÑOS PARA LOS MARCOS DÚCTILES SIN CONTRAVIENTOS

\begin{tabular}{|c|c|c|c|c|c|c|c|c|c|}
\hline MODELO & $\triangle M A X(\%)$ & $\begin{array}{r}\rho+V I G A S \\
(\%)\end{array}$ & $\begin{array}{r}\rho \text {-VIGAS } \\
(\%)\end{array}$ & $\begin{array}{r}\rho \text { COLUMNAS } \\
(\%)\end{array}$ & MODELO & $\triangle \mathrm{MAX}(\%)$ & $\begin{array}{r}\rho+V I G A S \\
(\%)\end{array}$ & $\begin{array}{r}\rho \text {-VIGAS } \\
(\%)\end{array}$ & $\begin{array}{r}\rho \text { COLUMNAS } \\
(\%)\end{array}$ \\
\hline M1-4LC & 1.65 & $0.81-1.10$ & $0.41-0.58$ & $1.0-1.3$ & M1-12LC & 2.8 & $1.10-1.25$ & $0.44-0.76$ & $1.2-1.5$ \\
\hline M2-4LC & 1.3 & $0.59-0.69$ & $0.33-0.35$ & $1.2-1.4$ & M2-12LC & 1.85 & $1.01-1.19$ & $0.79-0.95$ & $1.2-1.3$ \\
\hline M3-4LC & 0.9 & $0.46-0.63$ & $0.32-0.42$ & 1.3 & M3-12LC & 1.8 & $0.95-1.09$ & $0.87-0.99$ & $1.2-1.5$ \\
\hline M4-4LC & 0.9 & $0.64-0.73$ & $0.52-0.58$ & 1.3 & M4-12LC & 1.2 & 0.97-1.17 & $0.93-1.13$ & $1.2-1.5$ \\
\hline M1-8LC & 2.5 & $0.94-1.18$ & $0.48-0.66$ & $1.0-1.3$ & M1-16LC & 2.95 & $1.00-1.24$ & $0.54-0.89$ & $1.2-1.3$ \\
\hline M2-8LC & 1.4 & $0.76-0.92$ & $0.42-0.58$ & $1.0-1.3$ & M2-16LC & 2.2 & $1.09-1.29$ & $0.88-1.21$ & $1.0-1.5$ \\
\hline M3-8LC & 1.2 & $0.76-1.03$ & $0.51-0.85$ & $1.2-1.3$ & M3-16LC & 1.75 & $0.93-1.22$ & $0.83-1.19$ & $1.2-1.6$ \\
\hline M4-8LC & 1.2 & $0.79-1.04$ & $0.69-0.92$ & $1.2-1.3$ & M4-16LC & 1.35 & $0.92-1.22$ & $0.92-1.12$ & $1.2-2.2$ \\
\hline
\end{tabular}

de vigas y columnas, donde se aprecia que, en efecto, se tuvo el cuidado que estas fueran $\mathrm{ra}^{-}$ zonables (menores o cercanas al $1 \%$ a tensión en vigas y lo más cercanas al $1 \%-1.5 \%$ en columnas) para garantizar un comportamiento dúctil de dichos elementos. En la tabla 2 se reportan los periodos fundamentales elásticos de los modelos en estudio.

TABLA 2. PERIODOS FUNDAMENTALES ELÁSTICOS DE LOS MARCOS DÚCTILES NO CONTRAVENTEADOS

\begin{tabular}{|c|c|c|c|c|c|c|c|}
\hline MODELO & $\mathrm{T}$ (S) & MODELO & $\mathrm{T}(\mathbf{S})$ & MODELO & $\mathrm{T}(\mathbf{S )}$ & MODELO & $T(S)$ \\
\hline M1-4LC & 0.67 & M1-8LC & 1.138 & M1-12LC & 1.46 & M1-16LC & 1.608 \\
\hline M2-4LC & 0.592 & M2-8LC & 0.882 & M2-12LC & 1.223 & M2-16LC & 1.446 \\
\hline M3-4LC & 0.509 & M3-8LC & 0.828 & M3-12LC & 1.079 & M3-16LC & 1.264 \\
\hline M4-4LC & 0.516 & ML-8LC & 0.8 & M4-12LC & 1.007 & M4-16LC & 1.14 \\
\hline
\end{tabular}

Para el diseño de los elementos sismorresistentes de los marcos dúctiles con contravientos se empleó una metodología basada en conceptos de diseño por capacidad, donde los elementos estructurales se diseñan por resistencia del

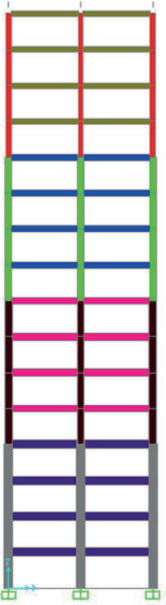

a) 16 niveles

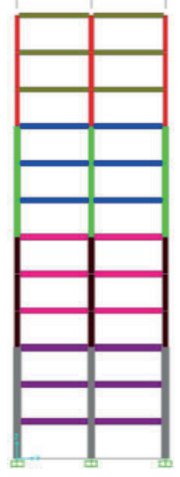

a) 12 niveles más débil al más fuerte, y con esto fomentar un mecanismo de colapso columna fuerte - viga débil - contraviento más débil. La secuencia de diseño empleada fue: 1) contravientos, 2) vigas, 3) columnas y, 4) conexiones. Asimismo, se emplearon las recomendaciones de diseño derivadas de una serie de análisis estáticos y dinámicos no lineales ante carga monótona creciente de marcos planos de entre cuatro y 24 niveles, los cuales se describen con detalle en Godínez (2010), Godínez-Domínguez y TenaColunga (2010) y Godínez et al. (2012). Por lo anterior, para el cálculo de los factores de reducción por sobrerresistencia (R, ec. 8), los límites de distorsión asociados al estado límite de servicio (ec. 9) y prevención de colapso (ec. 10, en este caso se considera la propuesta de las NTCS-04), así como los balances de resistencia (ec. 11) y consideraciones para el diseño a nivel local, se emplearon las recomendaciones derivadas de los estudios de referencia.

$$
R= \begin{cases}1.7+5.8\left(1-\sqrt{T e / T_{a}}\right) & \text { si } T_{e} \leq T_{a} \\ 1.7 ; & \text { si } T_{e}>T_{a}\end{cases}
$$

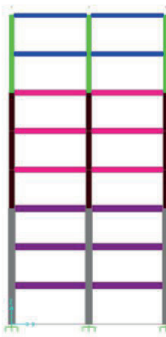

a) 8 niveles

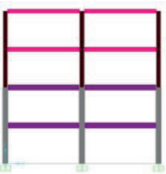

a) 14 niveles

Figura 8. Cambios de secciones transversales de vigas y columnas considerados en los modelos de marcos dúctiles no contraventeados, indicados esquemáticamente con el cambio de colores. 


$$
\begin{aligned}
& \Delta_{y}=0.002 \\
& \Delta_{\max -N T C S-04=0.015} \\
& V_{R C o l} \geq\left\{\begin{array}{cc}
50 & \text { si } \frac{H}{L} \leq 0.5 \\
50+10 \sqrt{\frac{H}{L}} & \text { si } 0.5<\frac{H}{L} \leq 4
\end{array}\right.
\end{aligned}
$$

dondE $\mathrm{T}_{\mathrm{a}} \mathrm{y}$ Te SON El periodo característico que define el inicio de la meseta en el espectro de diseño elástico y el periodo fundamental elástico de la estructura, $\Delta_{\mathrm{y}}$ es la distorsión de fluencia considerada en la revisión estado límite de servicio, $\Delta_{\max }$ es la distorsión máxima de diseño considerada en la revisión del estado límite de colapso, $V_{\mathrm{Rcol}}$ representa la aportación mínima de las columnas al cortante resistente ante carga lateral (en porcentaje), $\mathrm{H}$ es la altura total del edificio y $L$ la longitud de la base de la estructura en el sentido de análisis.

El criterio empleado para la definición del porcentaje de la resistencia a fuerza cortante total que el sistema de contraventeo debe ser capaz de aportar se realizó con base en la propuesta hecha en estudios previos de los autores (Godínez-Domínguez y Tena-Colunga 2010), donde se presenta una ecuación simple en función de la relación de esbeltez de la estructura (H/L), para la estimación del porcentaje mínimo de resistencia a fuerza cortante que las columnas de un entrepiso deben ser capaces de aportar en el sistema dual (ec. 11). Con lo anterior se busca que los mecanismos de colapso obtenidos sean congruentes con la filosofía de diseño, es decir, columnas fuerte-viga débilcontraviento más débil. La propuesta es congruente con la recomendación de las NTCS-04, en que se especifica que para el diseño de estructuras dúctiles en que la resistencia en todos los entrepisos es suministrada por marcos contraventeados, se requiere que en cada entrepiso los marcos sean capaces de resistir, sin contar con contravientos, cuando menos 50 por ciento de la fuerza sísmica actuante. Cabe señalar que la propuesta de las NTCS-04 es independil de esbeltez de la estructura considerada.

En los diseños realizados, la sección de columnas se varió de acuerdo al número de niveles del marco. En el caso de los marcos de cuatro niveles, la sección de columnas permaneció uniforme en altura, al igual que las vigas y contravientos. Para los marcos de 8 a 16 niveles, la variación de secciones de columnas y vigas se realizó cada cuatro niveles, alternando la variación de las secciones en el caso de los contravientos, en los que, en general, la variación del espesor de la sección quedó determinada de tal manera que las secciones propuestas estuviesen diseñadas lo más justas posible, pues teóricamente estos elementos deben representar la parte más débil del sistema estructural de acuerdo al mecanismo de colapso deseado.

Lo estrategia seguida para la variación de las secciones en elevación obedece a tratar de evitar crear planos de debilidad en algún entrepiso, pues se observó, por medio de análisis estáticos no lineales, que la práctica de variar en el mismo entrepiso las secciones de vigas, columnas y contravientos conducía a la posible generación de pisos débiles, ocasionando fallas no deseadas y reduciendo significativamente la ductilidad del sistema (Godínez 2010).

El dimensionamiento de vigas y columnas, así como el de los contravientos metálicos, se basó en los balances de rigidez y resistencia entre el marco y el sistema de contraventeo que deben cumplir para fomentar un comportamiento dúctil (Godínez 2010). Por lo anterior, en varios casos, los armados por flexión de vigas y columnas están asociados a cuantías mínimas, ya que se tienen secciones más robustas de lo requerido por resistencia. El refuerzo transversal se diseñó cumpliendo los criterios de resistencia por fuerza cortante y de confinamiento.

En todos los casos los contravientos son secciones cajón de acero A-36, como es práctica común tanto en el caso de proyectos de refuerzo estructural (concreto o acero), como para el caso de diseños nuevos de estructuras metálicas.

En la tabla 3 se resumen las propiedades dinámicas de cada modelo, así como la masa asociada al primer modo de vibrar.

TABLA 3. PERIODOS FUNDAMENTALES ELÁSTICOS DE LOS MARCOS
DÚCTILES CON CONTRAVIENTOS
\begin{tabular}{|l|c|c|c|c|}
\hline MODELO & T (S) MODELO & T (S) MODELO & T (S) MODELO & T (S) \\
\hline M1C4N & 0.286 M1C8N & 0.561 M1C12N & 0.81 M1C16N & 1.164 \\
\hline M2C4N & 0.302 M2C8N & 0.572 M2C12N & 0.789 M2C16N & 1.064 \\
\hline M3C4N & 0.348 M3C8N & 0.629 M3C12N & 0.898 M3C16N & 1.179 \\
\hline M4C4N & 0.361 M4C8N & 0.644 M4C12N & 1.027 M4C16N & 1.285 \\
\hline M3C4N-V1 & 0.274 M3C8N-V1 & 0.518 M3C12N-V1 & 0.779 M3C16N-V1 & 1.06 \\
\hline M4C4N-V1 & 0.295 M4C8N-V1 & 0.542 M4C12N-V1 & 0.82 M4C16N-V1 & 1.161 \\
\hline
\end{tabular}

ANÁLISIS NO LINEALES

Se realizaron análisis no lineales ante carga monótona creciente (pushover) con el programa DRAIN-2DX (Prakash et al. 1992). En el modelado de los marcos se hicieron las mismas consideraciones que en el modelado elástico, que fueron las siguientes: 1) inercias brutas para definir las 
rigideces elásticas, 2) 50\% del nudo viga-columna se modeló como zona de rigidez infinita, 3) condición de empotramiento perfecto en la base, 4) las cargas gravitacionales fueron incluidas, 5) el sistema de piso se comporta como un diafragma rígido y, 6) se utilizaron los mismos patrones de cargas laterales que se emplearon para realizar los diseños. Se incluyen en todos los casos los efectos P- $\Delta$ en columnas y diagonales y se ignora la interacción suelo estructura.

Para el modelado no lineal de los marcos se incluye el comportamiento no lineal de los elementos estructurales a nivel local. En el caso de vigas, se tomó en cuenta la aportación de la losa a su resistencia, rigidez y capacidad de deformación. Las propiedades estructurales de las vigas se derivaron de diagramas momento-curvatura empleando el programa Biax (Wallace y Moehle 1989), y corresponden a las secciones transversales ubicadas en sus extremos. La curvatura de fluencia corresponde a la primera cedencia del acero longitudinal, mientras que la curvatura última corresponde a la menor curvatura asociada a la fractura del acero longitudinal o al aplastamiento del concreto. Para el modelado de las columnas, sus propiedades estructurales se derivaron de diagramas de interacción carga axial-momento flexionante determinados también con el programa Biax.

En la definición de las superficies de falla de todos los modelos en estudio, se determinaron las resistencias a flexión de las trabes y a flexocompresión y flexotensión de las columnas de cada modelo considerando:

- El área real provista de refuerzo, incluyendo la aportación del refuerzo a compresión de las vigas.

- La curva esfuerzo-deformación «real» del concreto reforzado utilizando el modelo de Kent y Park modificado para tomar en cuenta el confinamiento provisto (Park et al. 1982).

- La curva esfuerzo-deformación real del acero de refuerzo empleando el modelo de Rodríguez y Botero (1995) para aceros de refuerzo mexicanos.

- El patín equivalente de la losa según NTCC-2004 así como el acero de refuerzo contenido dentro de esta porción de losa, al ser diseñadas de inicio las vigas como $\mathrm{T}$.

En la determinación de la sobrerresistencia de los contravientos, se consideró que el esfuerzo de fluencia que pueden desarrollar es mayor que su esfuerzo nominal de fluencia a tensión, y con base en la información disponible en la literatura para acero A-36 (Bruneau et al. 1998), este esfuerzo esperadodefluenciaes $f_{\text {y-espera }}=52 \mathrm{ksi}\left(3670 \mathrm{~kg} / \mathrm{cm}^{2}\right)$. Este valor de $f_{\text {y-esperado }}$ se utilizó para determinar también la carga de pandeo de los contravientos conforme a lo establecido en las NTCEM-04. Aquí cabe señalar que cuando se aumenta el esfuerzo de fluencia $f_{y}$, aumenta el parámetro de esbeltez $(\lambda)$, por lo que en ocasiones, la resistencia de pandeo no aumenta significativamente.

La capacidad rotacional de las vigas y columnas se estimó a partir de considerar una longitud de articulación plástica igual a la mitad de su peralte. La determinación de las longitudes de pandeo de los contravientos se hizo con base en la propuesta de Kemp (1996), donde se propone una metodología para la determinación de la capacidad de rotación de elementos sujetos a compresión.

Los análisis estáticos no lineales ante carga monótona creciente se controlaron por incrementos de desplazamientos y para cada modelo en estudio, se dejaron correr hasta que la estructura presentaba inestabilidad numérica. Posteriormente, los resultados obtenidos fueron revisados para determinar si el nivel de deformación último de la estructura era realista. Esto se hizo monitoreando las rotaciones plásticas de las vigas y columnas con los diagramas momento-curvatura obtenidos a partir del software BIAX (Wallace y Moehle 1989). Se procesaron entonces los resultados de los análisis no lineales considerando solamente hasta el paso de integración (incremento de desplazamiento) donde se asegura que las rotaciones plásticas obtenidas no excedieran en demasía las rotaciones plásticas máximas calculadas para cada sección en función de su geometría, armado y detallado, es decir, se cortaron (truncaron) algunos puntos calculados numéricamente, pero que ya no eran congruentes con el modelo estudiado. Se verificó entonces que el mecanismo de colapso exhibido por la estructura fuera dominantemente del tipo columna fuerte-viga débil, y entonces se trazaron las curvas de capacidad global de la estructura (cortante basal vs distorsión entre la base y la azotea) además, de sus curvas globales primarias (idealizaciones bilineales de las curvas obtenidas del análisis).

Asimismo, en los marcos con contravientos también se determinó la magnitud del acortamiento que pueden sufrir los contravientos al momento del pandeo (longitudes de pandeo), lo cual se hizo con base en la propuesta de Kemp (1996), la cual se apoya en pruebas experimentales realizadas por varios investigadores a nivel 
mundial. En general, el análisis se detuvo cuando la primera línea de defensa del sistema dual, constituida por el sistema de contraventeo había fallado, es decir, la mayoría de los contravientos en compresión alcanzaban su longitud de pandeo y la mayoría de las vigas de dos entrepisos consecutivos experimentaban demandas de rotación ligeramente superiores a las definidas por su capacidad teórica, además se cuidó la relación demanda-capacidad de las columnas, principalmente las ubicadas en planta baja.

\section{CURVAS GLOBALES CORTANTE-DISTORSIÓN} NORMALIZADAS

Se obtuvieron curvas globales de capacidad (cortante basal vs distorsión global base-azotea) para cada modelo (por ejemplo, Tena y Cortés 2014 y 2015, Godínez y Tena 2015), de las cuales se observó claramente que la rigidez de los sistemas se incrementa al aumentar el número de

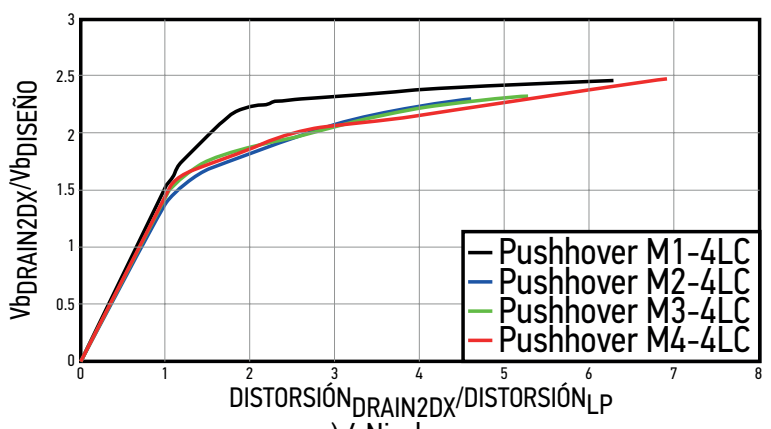

a) 4 Niveles

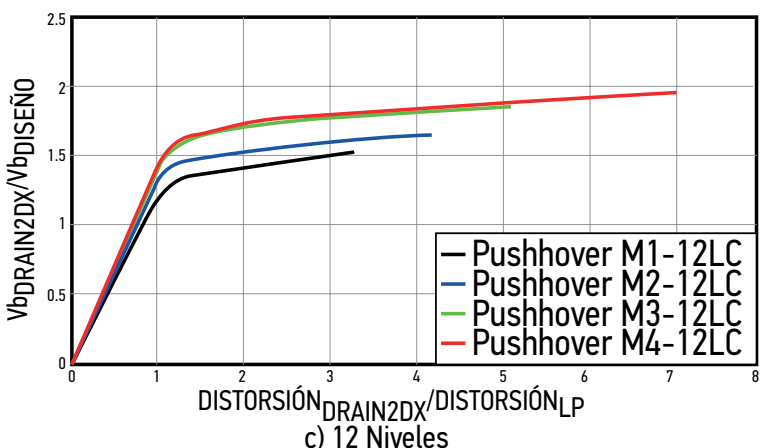

c) 12 Niveles líneas de defensa (crujías), y que presentadas de esta manera es difícil evaluar cualitativamente el impacto de la redundancia y la altura de las estructuras en su capacidad relativa de deformación (ductilidad). Por ello, se decidió normalizar a las curvas globales de capacidad de la siguiente manera: las abscisas (distorsiones) se normalizaron con respecto a la distorsión de la primera fluencia o donde termina el límite proporcional teóricamente $(\Delta y)$, y las ordenadas (cortante basal) se normalizaron con respecto al cortante basal de diseño. Con esta doble normalización es más fácil comparar el comportamiento entre estructuras de una misma altura, así como entre estructuras de diferentes alturas y, con ello, valorar el impacto de la redundancia tanto en la ductilidad como en la resistencia. En la figura 9 se presentan las curvas globales de capacidad normalizadas para los marcos dúctiles no contraventeados y en la figura 10 para los marcos dúctiles con contravientos.

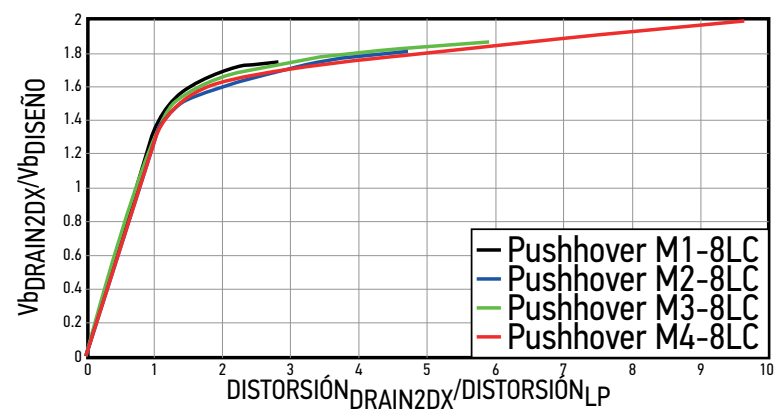

b) 16 Niveles

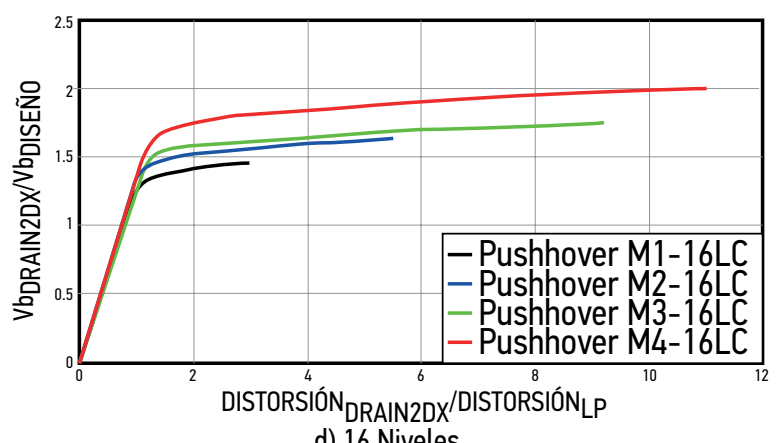

d) 16 Niveles

Figura 9. Curvas globales normalizadas cortante-distorsión para los marcos dúctiles no contraventeados

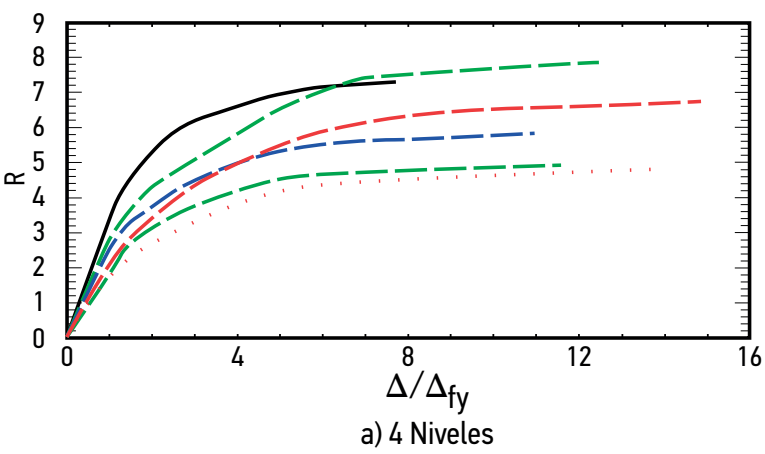

a) 4 Niveles

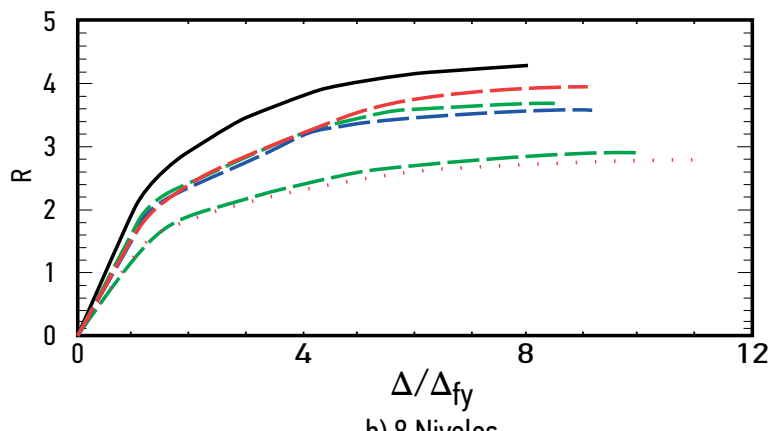

b) 8 Niveles 


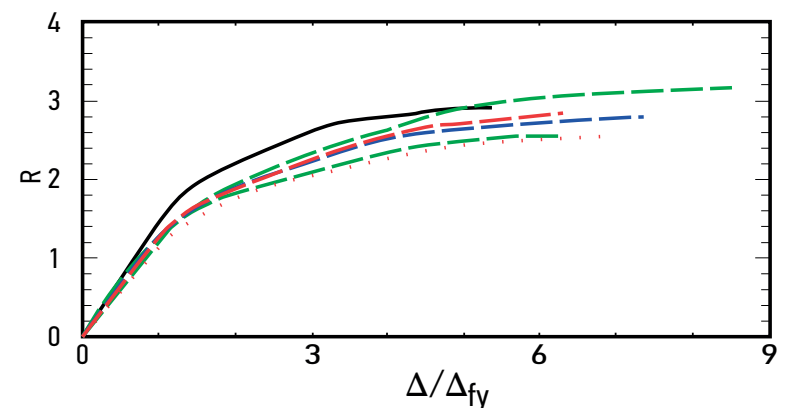

c) 12 Niveles

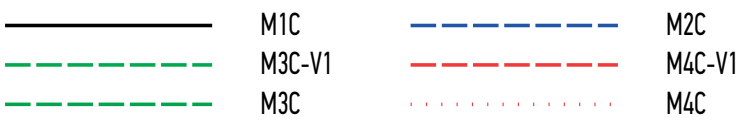

A partir de las curvas mostradas en las figura $9 y$ 10 se calcularon las ductilidades y sobrerresistencias desarrolladas, como se reporta con detalle en Tena y Cortés (2014 y 2015) para los marcos dúctiles no contraventeados y en Godínez y Tena (2015) para los marcos dúctiles con contravientos y que por limitaciones de espacio no se presentan en este trabajo.

\section{DETERMINACIÓN DEL FACTOR POR REDUNDANCIA}

Los resultados obtenidos de los análisis estáticos no lineales muestran que el efecto de aumentar la redundancia estructural impacta tanto en la resistencia como en la capacidad de deformación de los modelos. En el MOC-2008 (2009)

ya se toma en cuenta este efecto, al considerar que la redundancia repercute en la resistencia y ductilidad global de la estructura, reduciendo los espectros de diseño en función de Q', R y $\rho$, e incrementando las deformaciones obtenidas del análisis elástico multiplicándolas por $\mathrm{Q} R$ y $\rho$ (figura 5).

Por lo tanto, para evaluar el efecto de la redundancia con la altura de los modelos, se obtuvieron dos factores de redundancia: $\rho_{C D}$, que toma en cuenta el aumento en la capacidad de deformación, y $\rho_{\mathrm{R}}$, que toma en cuenta el aumento de la resistencia. Para definir estos dos factores de redundancia $\left(\rho_{\mathrm{CD}}\right.$ y $\left.\rho_{\mathrm{R}}\right)$, se tomaron como punto de partida la propuesta actual del MOC-2008 (2009), asignando entonces un valor de $\rho_{C D}$ y $\rho_{\mathrm{R}}$ igual uno a los modelos que tienen dos crujías en la dirección del análisis.

Para obtener el factor de redundancia $\rho_{\mathrm{R}}$, se normalizaron las sobrerresistencias de los modelos de una, tres y cuatro crujías en función de la sobrerresistencia que desarrolló el modelo de dos crujías:

$\rho_{R i}=\frac{R(\text { sobrerresistencia de los mod elos de una, tre o cuatro crujüas })}{R(\text { Sobrerresistencia del mod elo de dos crujuas })}$

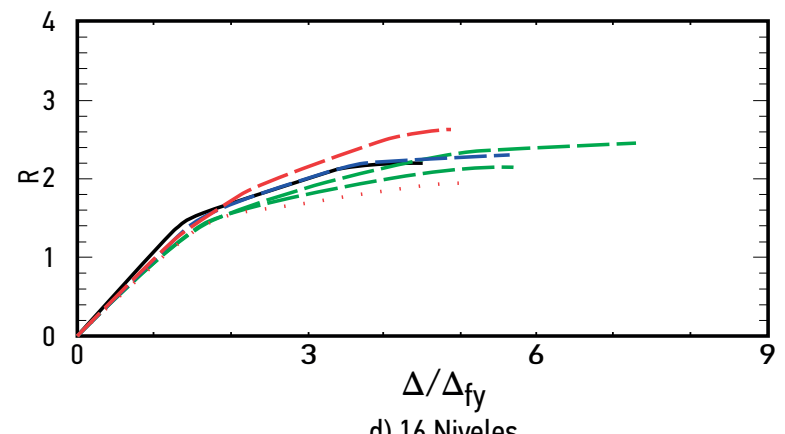

d) 16 Niveles

Figura 10. Curvas globales normalizadas cortante-distorsión para los marcos dúctiles con contravientos $\left(\mathrm{R}=\mathrm{V} / \mathrm{V}_{\mathrm{dis}}\right)$.

Para obtener el factor de redundancia $\rho_{\mathrm{CD}}$, se normalizaron las ductilidades de los modelos de una, dos y tres crujías en función de la ductilidad que desarrolló el modelo de dos crujías:

$\rho_{C D i}=\frac{Q \text { (Ductilidad de los mod elos de una, tres o cuatro crujias })}{Q(\text { Ductilidad del mod elo de dos crujias })}$

(13)

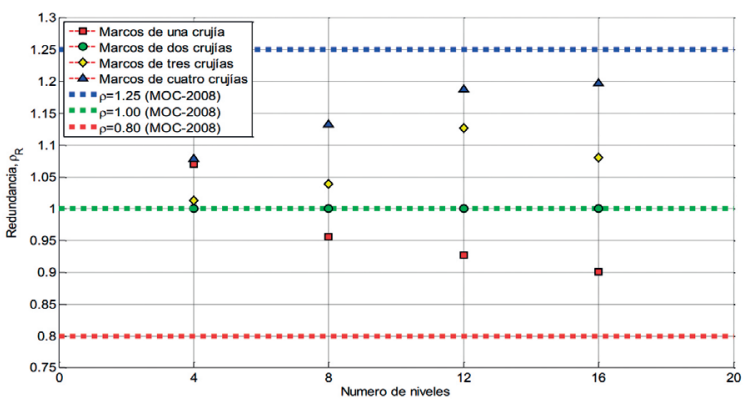

a) Factor $\rho$ R

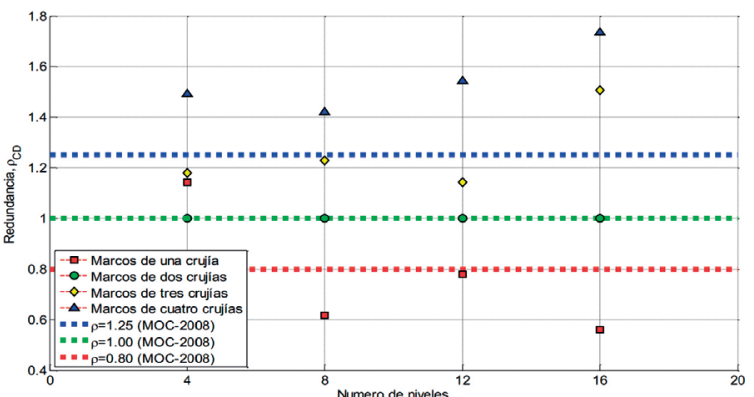

b) Factor $\rho C D$

Figura 11. Factores de redundancia $\rho R$ y $\rho C D$ calculados para los marcos dúctiles no contraventeados.

En la figura 11a se presenta el factor de reducción por redundancia $\rho R$ calculado para los marcos dúctiles no contraventeados. Se observa de dicha figura que el factor de redundancia $\rho R$ se incrementa en los modelos de tres y cuatro crujías, mientras que en los modelos de una crujía, disminuye al aumentar la altura de éstos. Con excepción del modelo de una crujía y cuatro niveles, el comportamiento obtenido en los modelos es el que se esperaba, que en los 
modelos de tres y cuatro crujías los factores de reducción por redundancia fueran mayores a uno, mientras que en los modelos de una crujía estos factores deberían ser menores a la unidad.

Si se comparan los valores obtenidos para $\rho_{R}$ calculado para los marcos no contraventeados (figura 11a) con la propuesta del MOC-2008, se aprecia que para los modelos de tres y cuatro crujías, a los cuales el MOC-2008 les asigna un factor de reducción por redundancia $\rho=1.25$, no alcanzan este valor. De hecho, quienes están más cerca a lograrlo son los modelos de cuatro crujías, donde $\rho_{R}$, se incrementa de 1.13 para los modelos de 8 niveles a 1.20 para los modelos de 16 niveles. Para los modelos de una crujía, en el MOC-2008 se propone castigar su diseño utilizando $\rho=0.8$. Sin embargo, se aprecia que para una crujía, $\rho_{\mathrm{R}}$ disminuye de 0.94 para los modelos de 8 niveles a 0.90 para los modelos de 16 niveles. Del análisis de los resultados presentados en la figura 11a se concluye que, para marcos dúctiles no contraventeados, el impacto de la redundancia estructural en la resistencia lateral es un poco menor a lo que se propone actualmente en el MOC-2008, pero que conceptualmente se está en el rumbo correcto.

En la figura 11b se presentan los factores de reducción por redundancia $\rho_{C D}$ calculados para marcos dúctiles no contraventeados, y se observa que el factor de redundancia $\rho_{C D}$ sigue el mismo patrón que el factor $\rho_{R}$, pero los efectos en cuanto a capacidad de deformación se refiere son mayores a los que se proponen en el MOC2008. Para los modelos de una crujía, el factor $\rho_{C D}$ calculado es menor al factor $\rho=0.8$ propuesto en el MOC-2008 para los modelos de más de 8 niveles, que son en los que dominaron en el diseño las combinaciones de cargas accidentales y gravitacionales y, por ende, son más representativos. Para los modelos de tres crujías se aprecia que el factor $\rho_{\mathrm{CD}}$ calculado es cercano o superior a $\rho=1.25$ propuesto en el MOC-2008, mientras que para los modelos de cuatro crujías el factor $\rho_{C D}$ calculado es siempre superior a $\rho=1.25$. En el MOC-2008 se propone utilizar un factor por reducción de redundancia igual a 1.25, mientras que el promedio de este factor de redundancia asociado a la capacidad de deformación de marcos dúctiles no contraventeados es de 1.41 para los modelos de tres y cuatro crujías.

En la figura 12a se presenta el factor de reducción por redundancia $\rho_{R}$ calculado para los marcos dúctiles con contravientos. Al comparar los valores del factor de redundancia $\rho_{R}$ respecto a los propuestos en el MOC-2008 (figura 12a), se aprecia que para los modelos de tres y cuatro crujías, a los cuales el MOC2008 les asigna un factor de reducción por redundancia $\rho=1.25$, únicamente en el modelo M3C4N-V1 se sobrepasa dicho valor $\left(\rho_{\mathrm{R}}=1.34\right)$, y en el resto de los casos $\rho_{R}$ alcanza como máximo un valor de 1.16 (modelo M4C16N-V1). Para los modelos de una crujía, en los que en el MOC-2008 se les asigna un valor $\rho=0.8$, se observó que los valores del factor $\rho_{R}$ decrecen al incrementarse el número de niveles, es decir, decrece conforme existe una mayor influencia de las combinaciones de cargas sísmicas en el diseño de los modelos. En este caso, el valor de $\rho_{R}$ decrece de 1.19 en el modelo de ocho niveles a un mínimo de 0.95 en el modelo de 16 niveles. Lo anterior indica que, para el caso de marcos dúctiles con contravientos metálicos tipo chevrón susceptibles a pandeo, el impacto de la redundancia estructural en la resistencia lateral es menor a lo que se propone actualmente en el MOC-2008. Si se comparan con los resultados obtenidos para marcos dúctiles sin contravientos (figura 11a), se aprecia que la tendencia es contraria, pues en los marcos dúctiles no contraventeados $\rho_{\mathrm{R}}$ tiende $\mathrm{a} \mathrm{au}^{-}$ mentar a medida que aumenta el número de crujías y niveles.

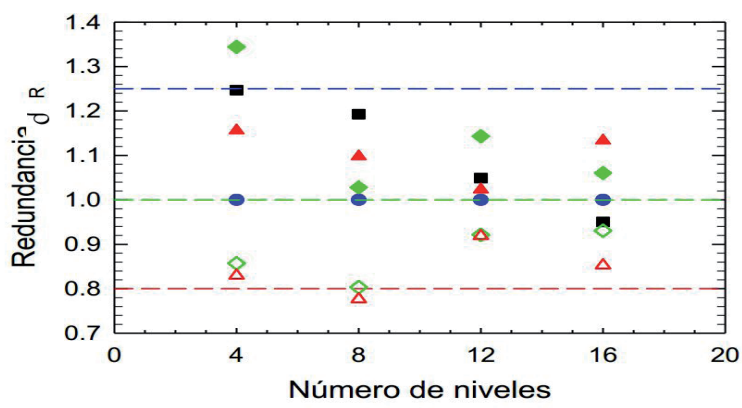

a) Factores de redundancia $\rho_{R}$

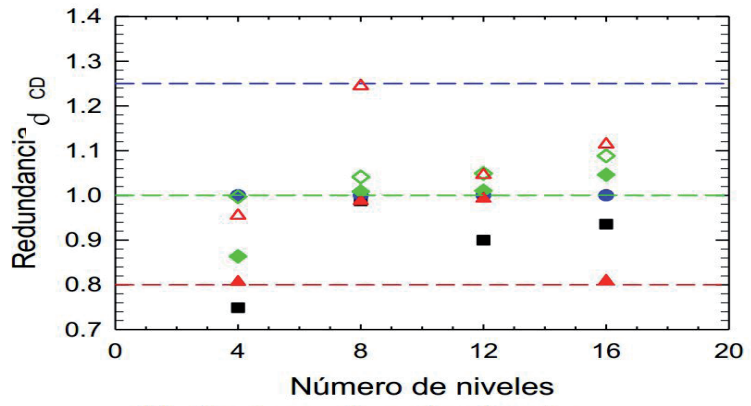

b) Factores de redundancia $\rho_{C D}$

- Marcos de una crujia Marcos de dos crujias

Marcos de tres crujias $\Delta \quad$ Marcos de cuatro crujias

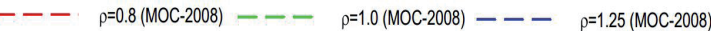

Figura 12. Factores de redundancia $\rho R$ y $\rho C D$ calculados para los marcos dúctiles contraventeados. 
En la figura $12 \mathrm{~b}$ se presentan los factores $\rho_{C D}$ calculados para marcos dúctiles contraventeados. Se observa que la variación de los factores $\rho_{C D}$ sigue la misma tendencia que los factores $\rho_{R}$ (figura 12a) para este sistema. Se aprecia de la figura $12 \mathrm{~b}$ que para los modelos de una crujía, el factor $\rho_{\mathrm{CD}}$ calculado es menor al factor $\rho=0.8$ propuesto en el MOC-2008 únicamente en los modelos de cuatro niveles $\left(\rho_{C D}=0.75\right)$, ya que a partir de los modelos de ocho niveles, que son en los que dominaron en el diseño las combinaciones de cargas accidentales y gravitacionales y, por ende, son más representativos, todos los valores son superiores a 0.8 , teniendo como valor promedio 0.94. Para los modelos de tres y cuatro crujías, como se esperaba, se aprecia que el factor $\rho_{C D}$ calculado es inferior a $\rho=1.25$ propuesto en el MOC-2008, teniendo como máximo un valor de 1.09 en los modelos de tres crujías y de 1.24 en los modelos de cuatro crujías. Dichos valores son inferiores a los obtenidos en marcos dúctiles de concreto reforzado no contraventeados (figura 11b). Lo anterior resulta lógico, pues los marcos dúctiles contraventeados constituyen un sistema estructural que es en general más resistente, pero menos dúctil que un sistema con base en marcos dúctiles a momento exclusivamente.

\section{CONCLUSIONES}

En presente artículo se resumen estudios en progreso sobre el impacto que tiene el aumentar la redundancia estructural en edificios con base en marcos dúctiles de concreto reforzado, con y sin contravientos, de distintos niveles, utilizando análisis no lineales con carga monótonamente creciente (pushover). Estos marcos fueron analizados bajo las recomendaciones del Apéndice A de las NTCS04, y diseñados como marcos dúctiles de concreto reforzado con las recomendaciones de las NTCC04. En los análisis no lineales se utilizó un modelado de plasticidad concentrada y se definió que los elementos vigas y columnas presentaran una no linealidad dominada por flexión. Con base en estas limitaciones y con los resultados de estos estudios iniciales se concluye lo siguiente.

- En cuanto al efecto de aumentar el número de crujías en las estructuras, se concluye que en estructuras de baja altura es menos significativo el proveerlas de una limitada redundancia estructural, pero toma importancia conforme la altura de los modelos aumenta. Esto se debe a que, para el coeficiente sísmico de diseño considerado $(\mathrm{c}=\mathrm{V} / \mathrm{W}=0.10)$, las combinaciones por carga vertical generalmente dominan los diseños para los marcos de poca altura, mientras que su importancia disminuye a medida que las estructuras tienen más niveles.

- Las hipótesis generales hechas en el MOC-2008, donde se considera que el aumento de la redundancia estructural aumenta tanto a la resistencia como a la capacidad de deformación de las estructuras, se cumplen razonablemente para los modelos donde las combinaciones de cargas accidentales con gravitaciones rigieron el diseño (8 a 16 niveles). Cuando las combinaciones de cargas gravitacionales rigieron el diseño de vigas (modelos de 4 niveles), no se observó lo mismo y, para ese tipo de estructuras, la redundancia estructural no tuvo el impacto esperado.

- Al analizar los factores de reducción por redundancia obtenidos en este estudio, se concluye que al parecer, para marcos dúctiles de concreto reforzado (con y sin contravientos), la redundancia estructural afecta de manera diferente a la resistencia y capacidad de deformación. Para marcos dúctiles no contraventeados, el impacto más importante de la redundancia es en la capacidad de deformación. En contraste, para marcos dúctiles con contravientos, el impacto más importante de la redundancia es en la sobrerresistencia que se desarrolla. En la propuesta actual del MOC-2008, se propone que la reducción por redundancia afecte de la misma manera la resistencia como la capacidad de deformación.

- También es importante recalcar que al parecer, para marcos dúctiles de concreto reforzado (con o son contravientos), a medida que aumenta el número de niveles de los edificios, los factores de reducción por redundancia tienden a dejar de variar y mantenerse constantes, es decir, a alcanzar un límite.

Con base en los resultados de los estudios en progreso y de estudios previos reportados en la literatura especializada, se concluye que, en aras de la transparencia en el diseño sismorresistente de marcos dúctiles de concreto reforzado (con o sin contravientos) y otros sistemas 
estructurales, sí se justifica que la redundancia estructural sea tomada en cuenta directamente en el diseño mediante un factor de reducción por redundancia $(\rho)$, como lo proponen y hacen normas estadounidenses como el ASCE-7 o IBC, y mexicanas como el Manual de Obras Civiles (MOC-2008). Se demuestra que la redundancia afecta de distinta manera la capacidad de deformación y de resistencia, dependiendo las características del sistema estructural empleado.

La propuesta actualmente contenida en el MOC-2008 está conceptualmente en el rumbo correcto, pero como se reconoce en los comentarios de ese documento: "aunque la propuesta se basa en los resultados de varios estudios, también está basada en la intuición y la experiencia, por lo que es susceptible de revisión y de mejoras que se verán reflejadas en futuras revisiones de este cuerpo normativo». Por ello, parece que la discusión sobre los ajustes necesarios a hacerse son, entre otros temas: (1) ¿se debe proponer $\rho$ con base en el mínimo valor obtenido entre resistencia ó capacidad de deformación, o un promedio ponderado?, (2) ise debe tomar en cuenta el posible dominio de cargas verticales en el diseño a la hora de

\section{REFERENCIAS BIBLIOGRÁFICAS}

1. ASCE 7-(2010), "Minimum design loads for buildings and other structures", ASCE Standard ASCE/SEI 710, American Society of Civil Engineers, ISBN 0-7844-0809-2.

2. Bertero, R. D. y V. V. Bertero (1999), "Redundancy in earthquake resistant design", ASCE Journal of Structural Engineering, Vol. 125, No. 1, pp. 81-88, enero.

3. Bruneau, M., C.-M. Uang y A. Whittaker (1998), Ductile design of steel structures, primera edición, McGraw-Hill.

4. Cortés, J. A. (2012), "Evaluación de factores de reducción por redundancia estructural en el diseño sísmico de marcos dúctiles de concreto reforzado", Tesis de Maestría, Posgrado en Ingeniería Estructural, División de Ciencias Básicas e Ingeniería, Universidad Autónoma Metropolitana Azcapotzalco, marzo.

5. CSI-2005 (2005), CSI analysis reference manual for SAP2000, ETABS, and SAFE, Computers and Structures, Inc., Berkeley, California, USA, octubre.

6. Fallah, A. A., A. S. Moghadam y S. Mohammadzadeh (2009)", "Reliability index for reinforced concrete frames using nonlinear pushover and dynamic analysis", Interna- definir $\rho$, como una excepción a la regla? y, (3) ¿se debe afinar cuál es el número de líneas de defensa mínimas para obtener el máximo valor de $\rho$ que establezca un reglamento? Estas son obviamente asignaturas pendientes que requieren de estudios adicionales que permitan aclarar el panorama en este sentido.

\section{AGRADECIMIENTOS}

Los dos primeros autores agradecen a la Dirección de Obras del Gobierno del Distrito Federal por su patrocinio parcial de esta investigación. El segundo autor agradece la beca otorgada por el Consejo Nacional de Ciencia y Tecnología de México (Conacyt), que le permitió involucrarse en este proyecto de investigación en el desarrollo de su tesis de maestría en el Posgrado en Ingeniería Estructural de la UAM Azcapotzalco. El tercer autor agradece el apoyo para la realización de este proyecto a la Secretaria de Educación Pública, mediante el programa para la incorporación de nuevos profesores de tiempo completo de la convocatoria de PROMEP 2013, mediante el convenio PROMEP/103.5/13/6999 y número de folio UNACH-PTC-136.

tional Journal of Advanced Structural Engineering, Vol. 1, No. 2, pp. 135-151.

7. Feng, Y. S. y F. Moses (1986), "Optimum design, redundancy, and reliability of structural systems", Computers and Structures, Vol. 24, No. 2, pp. 239-251.

8. Frangopol, D. M. y J. P. Curley (1987), "Effects of damage and redundancy on structural reliability", ASCE Journal of Structural Engineering, Vol. 113, No. 7, pp. 1533-1549.

9. Fu, G. y D. M. Frangopol (1990), "Balancing weight, system reliability and redundancy in a multiobjective optimization framework", Structural Safety, Vol. 7, No. 2-4, pp. 165-175.

10. Godínez, E.A. (2010), "Estudio del comportamiento de marcos dúctiles de concreto reforzado con contraventeo chevrón", Tesis de Doctorado, Posgrado en Ingeniería Estructural, División de Ciencias Básicas e Ingeniería, Universidad Autónoma Metropolitana, julio.

11. Godínez, E. A. y A. Tena (2015), "Redundancia estructural en marcos dúctiles de concreto reforzado con contraventeo metálico tipo chevrón", Revista Internacional de Ingeniería de Estructuras, Vol. 20, No. 1, pp. 41-70. 
12. Godínez-Domínguez, E. A. y A. Tena-Colunga (2010), "Nonlinear behavior of code-designed reinforced concrete concentric braced frames under lateral loading”, Engineering Structures, Vol. 32, pp. 944-963.

13. Godínez-Domínguez, E. A., A. Tena-Colunga y L. E. Pérez-Rocha (2012), "Case studies on the seismic behavior of reinforced concrete chevron braced framed buildings", Engineering Structures, Vol. 45, pp. 78-103.

14. Husain, M. y P. Tsopelas (2004), "Measures of structural redundancy in RC buildings. I: Redundancy Indices", ASCE Journal of Structural Engineering, Vol. 130, No. 11, pp. 1651-1658, noviembre.

15. Kemp, R. A. (1996), "Inelastic local and lateral buckling in design codes", ASCE Journal of Structural Engineering, Vol. 122, No. 4, abril, pp. 374-382.

16. MOC-2008 (2009), Manual de diseño de obras civiles, Diseño por sismo, Instituto de Investigaciones Eléctricas, Comisión Federal de Electricidad, noviembre.

17. NTCCA-04 (2004), "Normas Técnicas Complementarias sobre Criterios y Acciones para el Diseño Estructural de las Edificaciones”, Gaceta Oficial del Distrito Federal, Tomo II, No. 103-BIS, octubre.

18. NTCC-04 (2004), "Normas Técnicas Complementarias para Diseño de Estructuras de Concreto", Gaceta Oficial del Distrito Federal, Tomo II, No. 103-BIS, octubre.

19. NTCS-04 (2004), "Normas Técnicas Complementarias para Diseño por Sismo", Gaceta Oficial del Distrito Federal, Tomo II, No. 103-BIS, octubre.

20. Paliou, C., M. Shinozuka y Y.-N. Chen (1990), "Reliability and redundancy of offshore structures", ASCE Journal of Engineering Mechanics, Vol. 116, No. 2, pp. 359-378.

21. Park, R., M. J. N. Priestley y W. D. Cill (1982), "Ductility of square-confined concrete columns", ASCE Journal of Structural Engineering, Vol. 108, No. 4, pp. 929-950.

22. Prakash, V., G. H. Powell y F. Fillipou (1992), "DRAIN-2DX: Base program user guide", Report No. UBC/SEMM-92/29, Department of Civil Engineering, University of California at Berkeley.

23. Rodríguez, M. y J. C. Botero (1995), "Comportamiento sísmico de estructuras considerando propiedades mecánicas de aceros de refuerzo mexicanos", Revista de Ingeniería Sísmica, SMIS, No. 49, pp. 39-50.
24. Song, S.H. y Y. K. Wen (2000), "Structural redundancy of dual and steel moment frame systems under seismic excitation", Structural Research Series No. 631, Department of Civil Engineering, University of Illinois at Urbana-Champaign, November.

25. Tena, A. (2010), "Irregularidad estructural y su efecto en la respuesta sísmica de edificios", Memorias, V Congreso Iberoamericano de Ingeniería Civil en honor del Dr. José Grases, Mérida, Venezuela, CD-ROM pp. 1-57, noviembre.

26. Tena, A. y J. A. Cortés (2014), "Impacto de la redundancia estructural en el comportamiento sísmico de marcos dúctiles de concreto reforzado", Revista Internacional de Ingeniería de Estructuras, Vol. 19, No. 1, pp. 1-34.

27. Tena, A., G. Gatica, L. A. Urbina y L. Victoria (2012), "Impacto de la disposición de vigas secundarias en losas perimetralmente apoyadas en el comportamiento sísmico de edificios diseñados con base en marcos de concreto reforzado", Revista Internacional de Ingeniería de Estructuras, Vol. 17, No. 1 y 2, pp. 63-85.

28. Tena-Colunga, A. (2004), "Evaluation of the seismic response of slender, setback RC moment-resisting frame buildings designed according to the seismic guidelines of a modern building code ",Memorias, 13th World Conference on Earthquake Engineering, Vancouver, Canada, Artículo No. 2027, CD-ROM, agosto.

29. Tena-Colunga, A., H. Correa-Arizmendi, J. L. Luna-Arroyo y G. Gatica-Avilés (2008), "Seismic Behavior of code-designed medium rise special moment-resisting frame RC buildings in soft soils of Mexico City", EngineeringStructures, Vol. 30, No. 12, pp. 36813707, doi:10.1016/j.engstruct.2008.05.026.

30. Tena-Colunga, A., U. Mena-Hernández, L. E. Pérez-Rocha, J. Avilés, M. Ordaz y J. I. Vilar (2009), "Updated seismic design guidelines for buildings of a model code of Mexico", Earthquake Spectra, Vol. 25, No. 4, pp. 869898, noviembre, doi: 10.1193/1.3240413.

31. Tena-Colunga, A. y J. A. Cortés-Benítez (2015), "Assessment of redundancy factors for the seismic design of special moment resisting reinforced concrete frames", Latin American Journal of Solids and Structures, Vol. 12, No. 12 , pp. 2330-2350.

32. Tsopelas, P. y M. Husain (2004), "Measures of structural redundancy in RC 
buildings. II: Redundancy response modification factor (RR)", ASCE Journal of Structural Engineering, Vol. 130, No. 11, pp. 16591666, noviembre.

33. UBC-97 (1997), Uniform Building Code, 1997 edition, International Conference of Building Officials, Whittier, California, Vol. 2.

34. Wallace, J. y J. Moehle (1989), "BIAX: A computer program for the analysis reinforced concrete sections" Report No. UCB/SEMM89/12, Department of Civil Engineering, University of California at Berkeley.
35. Web Berkeley (2010), Página web http://nisee. berkeley.edu/images/servlet/EqiisListQuake, cortesía de National Information Service for Earthquake Engineering (NISEE) de la Universidad de California en Berkeley. Las fotos corresponden a la colección del profesor Karl Steinbrugge.

36. Whittaker, A., G. Hart y C. Rojahn (1999), "Seismic response modification factors", ASCE Journal of Structural Engineering, Vol. 125, No. 4, pp. 438-444. 